\title{
Exertional dyspnoea in cardiorespiratory disorders: the clinical use of cardiopulmonary exercise testing
}

\author{
Pierantonio Laveneziana ${ }^{1,2}$ and Piergiuseppe Agostoni ${ }^{3,4}$
}

\begin{abstract}
Affiliations: 'Sorbonne Universités, UPMC Université Paris 06, INSERM, UMRS 1158 Neurophysiologie respiratoire expérimentale et clinique, Paris, France. ${ }^{2}$ Assistance Publique-Hôpitaux de Paris, Groupe Hospitalier Pitié-Salpêtrière Charles Foix, Service des Explorations Fonctionnelles de la Respiration, de l'Exercice et de la Dyspnée (Département "R3S", Pôle PRAGUES), Paris, France. ${ }^{3}$ Centro Cardiologico Monzino, IRCCS, Milan, Italy. "Dept of Clinical Sciences and Community Health, Cardiovascular Section, University of Milan, Milan, Italy.

Correspondence: Pierantonio Laveneziana, Service d'Explorations Fonctionnelles de la Respiration, de l'Exercice et de la Dyspnée, Département “R3S” (Respiration, Réanimation, Réhabilitation, Sommeil), Pôle PRAGUES, Hôpital Universitaire Pitié-Salpêtrière, Assistance Publique-Hôpitaux de Paris (AP-HP), 47-83 Boulevard de l'Hôpital, 75013, Paris, France. E-mail: pierantonio.lavenezianađaphp.fr
\end{abstract}

@ERSpublications

A series of articles on exertional dyspnoea in cardiorespiratory disorders begins in the European Respiratory Review http://ow.ly/fLT0300uHgw

Is dyspnoea worth documenting and accurately assessing? The answer is undoubtedly yes, for at least the following reasons. 1) Activity-related dyspnoea is usually the earliest and most troublesome complaint for which patients with cardiopulmonary diseases seek medical attention. 2) This symptom progresses relentlessly as the underlying disease advances, leading invariably to avoidance of activity with consequent skeletal muscle deconditioning and an impoverished quality of life. 3) It is estimated that up to a quarter of the general population and half of severely ill patients are affected by it. 4) Dyspnoea is also an important predictor of quality of life, exercise tolerance and mortality in various conditions. In patients with chronic obstructive pulmonary disease (COPD), it has been shown to be a better predictor of mortality than forced expiratory volume in $1 \mathrm{~s}$. In patients with heart disease referred for clinical exercise testing, it is a better predictor of mortality than angina. 5) Dyspnoea is also associated with decreased functional status and worse psychological health in older individuals living at home. 6) It is also a factor in the low adherence to exercise training programmes in sedentary adults and in patients with COPD. 7) The effective management of exertional dyspnoea remains a major challenge for caregivers and modern treatment strategies that are based on attempts to reverse the underlying chronic condition are only partially successful [1-7].

The perception of dyspnoea involves the integration of afferent and efferent inputs at a cortical level and is modulated by affective, emotional and behavioural components. The recent statements of the American Thoracic Society (ATS) [8] and European Respiratory Society [1] have underlined the multidimensional nature of dyspnoea which comprises three major dimensions: 1) the sensory-perceptual domain, 2) the affective distress and 3) the symptom impact or burden.

Dyspnoea is a complex, multifaceted and highly personalised sensory experience, the source and mechanisms of which are incompletely understood: there is no unique central or peripheral source of this symptom. The definition given by the latest ATS statement ("a subjective experience of breathing discomfort that consists of qualitatively distinct sensations that vary in intensity") highlights the importance of the

Received: May 032016 | Accepted: May 092016

Conflict of interest: Disclosures can be found alongside this article at err.ersjournals.com

Provenance: Submitted article, peer reviewed.

Copyright OERS 2016. ERR articles are open access and distributed under the terms of the Creative Commons Attribution Non-Commercial Licence 4.0. 
different qualities (descriptors) covered by the term dyspnoea, the cortical integration of multiple sources of neural inputs about breathing, and the physiological, affective and behavioural consequences [8]. More specifically, it is believed that dyspnoea arises when there is a conscious awareness of dissociation between what the brain expects (through the "corollary discharge", i.e. copy of the descending motor activity to perceptual areas) and what it receives in terms of neural inputs from the respiratory system, respiratory muscles, peripheral chemoreceptors and locomotor muscles [8]. Of course, not all conscious breathing sensations are labelled as "dyspnoea" because the brain is able to "filter" all the respiratory sensations and let only some of them reach the conscious level [1]. This sensory gating process hinders the brain from being constantly overloaded by irrelevant sensory information. Human beings have the voluntary capacity to bring "breathing" into awareness at any moment. However, breathing may come to conscious awareness automatically if it needs to be attended to (gate-in). This "gating process" is instrumental in monitoring essential biological and physiological functions, and adopting appropriate behaviour. It has been proposed that respiratory sensations are the result of neural gating into the cerebral cortex of respiratory afferent input eliciting a somatosensory cognitive awareness of breathing and an affective response [1].

It can be summarised in a very simplistic yet paedagogic way that dyspnoea may result from two processes: 1) a discriminative one that identifies relevant afferent information on respiratory disruption/ abnormalities and brings them to consciousness (sensory components: intensity and quality); and 2) an affective one that labels the now conscious sensation as unpleasant or threatening, i.e. dyspnoea.

Dyspnoea is not a single sensation, and our brain is able to distinguish among different afferent sources of information and give each of them a distinct cognitive sensation, notably: 1) work/effort ("breathing requires work or effort"); 2) tightness ("chest is constricted" or "chest feels tight"); and 3) air hunger ("unsatisfied inspiration", "starved for air", "urge to breathe" or "like breath holding"). It is generally accepted that these sensations do not share the same physiological mechanisms $[1,8]$. Some of these sensations, such as work/effort and air hunger, may exist and vary independently in the same subject or experimental condition. Notwithstanding, whether there are multiple types of dyspnoea, whether they are a subjective expression of different neurophysiological mechanisms, or the same neurophysiological mechanisms may be expressed differently in relation to language, psychological, cultural and/or social context is, at the moment, unclear and required additional research.

Dyspnoea can also be quantified ("intensity"). Exertional dyspnoea can be easily defined as "the perception of respiratory discomfort that occurs for an activity level that does not normally lead to breathing discomfort". It follows that the intensity of dyspnoea can be determined by assessing the activity level required to produce dyspnoea (i.e. dyspnoea at rest is more severe than dyspnoea when climbing stairs) [1].

Dyspnoea can therefore be evaluated during a physical task, such as cardiopulmonary exercise testing (CPET) $[1,8]$. For this purpose, the 10-point Borg scale can be used to rate a specific respiratory sensation (e.g. inspiratory difficulty, breathing effort, expiratory difficulty or air hunger) or a more general one (e.g. breathing difficulty or breathlessness). The magnitude of respiratory sensation should be anchored at both extremes of the scale such that a rating of "0" represents no breathing discomfort and " 10 " represents the maximal breathlessness that the subject had ever experienced or could imagine experiencing. Though somewhat less popular, the visual analogue scale (VAS) is another dyspnoea-measuring instrument with proven construct validity used during CPET. Both the VAS and Borg scale have been shown to provide similar scores during CPET, and to be reliable and reproducible over time in healthy subjects, and in patients with chronic respiratory diseases undergoing CPET [9]. The advantage of using the Borg or VAS scales in individual patients is the possibility of reliably comparing "intensity of exertional dyspnoea" at the same level of exercise activity (standardised work-rate or oxygen consumption or ventilation during CPET) between subjects, and before and after a pharmacological and/or nonpharmacological treatment [9]. Studies in cardiopulmonary diseases have shown that during CPET, there is a close correlation between the magnitude (and duration) of respiratory effort (measured by tidal oesophageal pressure relative to maximum) and the intensity of dyspnoea (measured by the Borg scale), and that pharmacological manipulations able to reduce the magnitude (and duration) of respiratory effort are clearly and consistently associated with reduced dyspnoea intensity $[1,9]$.

Quality and quantity of dyspnoea can also be measured together during CPET. Few studies have shown that moderate-to-severe COPD and even mild stable asthmatics are able to "perceive" dynamic changes in their mechanics of breathing during exercise: once a critical inspiratory reserve volume (IRV) is attained $(0.3-0.5 \mathrm{~L}$ from total lung capacity), tidal volume expansion is critically constrained, dyspnoea intensity rises abruptly and there is a transition in the dominant qualitative descriptor choice from work/effort to difficult/unsatisfied inspiration [1]. The clinical relevance of these findings is that by asking the quality of dyspnoea at the end of an exercise bout one can ascertain whether a critical IRV (difficult/unsatisfied inspiration) or not (work/ effort) has been reached, i.e. the likelihood of developing exercise-related critical volume constraints [1]. 
Things are, however, somehow different in chronic heart failure. Indeed, heart failure patients may report dyspnoea alone or in variable combination with leg discomfort as the exercise-limiting symptoms. In heart failure, no haemodynamic and respiratory variables, either at rest or during exercise, seem to be able to discriminate between heart failure patients who complain of dyspnoea as the predominant exercise limiting symptom from those who report leg discomfort. Clearly the issue is still unsolved and more studies are needed [1].

CPET is therefore well suited for understanding mechanisms underlying dyspnoea during exercise (exertional dyspnoea) both in research and clinical settings. CPET may also help clinicians identify additional mechanisms underpinning the greater dyspnoea intensity that could be independent of or not directly related to the main/obvious pathophysiological determinant of the disease under consideration.

We hope we have convinced readers of the importance of assessing dyspnoea on exertion. A clinical series of articles starting in this issue of the European Respiratory Review [10,11] will therefore focus on the clinical use of CPET to explore and unmask the mechanisms underlying dyspnoea during exercise in an abroad spectrum of cardiorespiratory disorders, covering topics including exertional dyspnoea in COPD, chronic heart failure, asthma, obesity, pulmonary arterial hypertension and interstitial lung diseases.

\section{References}

1 Laviolette L, Laveneziana P. Dyspnoea: a multidimensional and multidisciplinary approach. Eur Respir J 2014; 43: $1750-1762$

2 Banzett RB, O’Donnell CR. Should we measure dyspnoea in everyone? Eur Respir J 2014; 43: 1547-1550.

3 Gronseth R, Vollmer WM, Hardie JA, et al. Predictors of dyspnoea prevalence: results from the BOLD study. Eur Respir J 2014; 43: 1610-1620.

4 Nishimura K, Izumi T, Tsukino M, et al. Dyspnea is a better predictor of 5-year survival than airway obstruction in patients with COPD. Chest 2002; 121: 1434-1440.

5 Abidov A, Rozanski A, Hachamovitch R, et al. Prognostic significance of dyspnea in patients referred for cardiac stress testing. N Engl J Med 2005; 353: 1889-1898.

6 Ho SF, O'Mahony MS, Steward JA, et al. Dyspnoea and quality of life in older people at home. Age Ageing 2001; 30: $155-159$.

7 Hayton C, Clark A, Olive S, et al. Barriers to pulmonary rehabilitation: characteristics that predict patient attendance and adherence. Respir Med 2013; 107: 401-407.

8 Parshall MB, Schwartzstein RM, Adams L, et al. An official American Thoracic Society statement: update on the mechanisms, assessment, and management of dyspnea. Am J Respir Crit Care Med 2012; 185: 435-452.

9 Puente-Maestu L, Palange P, Casaburi R, et al. Use of exercise testing in the evaluation of interventional efficacy: an official ERS statement. Eur Respir J 2016; 47: 429-460.

10 Dubé B-P, Agostoni P, Laveneziana P. Exertional dyspnoea in chronic heart failure: the role of the lung and respiratory mechanical factors. Eur Respir Rev 2016; 25: 317-332.

11 O'Donnell DE, Elbehairy AF, Faisal A, et al. Exertional dyspnoea in COPD: the clinical utility of cardiopulmonary exercise testing. Eur Respir Rev 2016; 25: 333-347. 